International Journal of Pure and Applied Mathematics

Volume $91 \quad$ No. 2 2014, 237-244

ISSN: 1311-8080 (printed version); ISSN: 1314-3395 (on-line version)

url: http://www.ijpam.eu

doi: http://dx.doi.org/10.12732/ijpam.v91i2.10

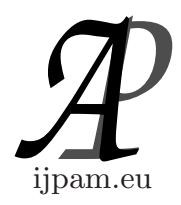

\title{
SOME PROPERTIES OF THE EULER SUMMABILITY METHOD IN COMPLETE ULTRAMETRIC FIELDS
}

\author{
R. Deepa ${ }^{1}$, K. Ganesan ${ }^{2}$ \\ ${ }^{1,2}$ Department of Mathematics \\ Faculty of Engineering and Technology \\ SRM University \\ Kattankulathur, Chennai, 603203, INDIA
}

\begin{abstract}
In this paper, we study some properties of the Euler method of summability in complete, non-trivially valued, ultrametric fields of characteristic zero and prove few Tauberian theorems of Euler method on such a field.
\end{abstract}

AMS Subject Classification: 40, 46

Key Words: complete ultrametric fields, regular summability methods, Euler method, translative methods, Tauberian theorem

\section{Introduction and Preliminaries}

Throughout the present paper, $K$ denotes a complete, non-trivially valued, ultrametric field of characteristic zero $\left(Q_{p}\right.$, the $p$-adic field for a prime $p$, is one such field). Infinite matrices, sequences and series considered in the sequel have entries in $K$. Given an infinite matrix $A=\left(a_{n k}\right), a_{n k} \in K, n, k=0,1,2, \cdots$ and a sequence $x=\left\{x_{k}\right\}, x_{k} \in K, \quad k=0,1,2, \ldots$, by the $A$-transform of $x=\left\{x_{k}\right\}$, we mean the sequence $A x=(A x)_{n}$, where $(A x)_{n}=\sum_{k=0}^{\infty} a_{n k} x_{k}, n=$ $0,1,2, \cdots$, it being assumed that the series on the right converge. If $\left\{(A x)_{n}\right\}$

Received: November 25, 2013

(C) 2014 Academic Publications, Ltd. url: www.acadpubl.eu

${ }^{\S}$ Correspondence author 
converges to $s$, we say that $x=\left\{x_{k}\right\}$ is summable $A$ or $A$-summable to $s$. If $\lim _{n \rightarrow \infty}(A x)_{n}=s$ whenever $\lim _{k \rightarrow \infty} x_{k}=s$, we say that $A$ is regular. The following theorem, which gives necessary and sufficient conditions for $A=\left(a_{n k}\right)$ to be regular in terms of the entries of the matrix, is well-known (see [4] for a proof using 'Uniform Boundedness Principle' and [5] for a proof using 'Sliding Hump Method').

Theorem 1.1. $A=\left(a_{n k}\right)$ is regular if and only if

(i) $\sup _{n, k}\left|a_{n k}\right|<\infty$;

(ii) $\lim _{n \rightarrow \infty} a_{n k}=0, k=0,1,2, \ldots$;and

(iii) $\lim _{n \rightarrow \infty} \sum_{k=0}^{\infty} a_{n k}=1$.

An infinite series $\sum_{k=0}^{\infty} x_{k}, x_{k} \in k, k=0,1,2, \cdots$ is said to be $A$-summable to $s$ if $\left\{s_{n}\right\}$ is $A$-summable to $s$, where $s_{n}=\sum_{k=0}^{n} x_{k}, n=$ $0,1,2, \cdots$.

In the present paper, we prove some interesting properties of the Euler method of summability introduced earlier by Natarajan [9].

General references for the study of summability methods in the classical case are [3], [10], while, for analysis in ultrametric fields, see [2].

Definition 1.1. Let $r \in K$ be such that $|1-r|<1$. The Euler method of order $r$ or the $(E, r)$ method is given by the infinite matrix $e_{n k}(r)$ which is defined as follows:

If $r \neq 1$, then $e_{n k}(r)=\left\{\begin{array}{l}{ }^{n} c_{k} r^{k}(1-r)^{n-k}, \quad \text { for } \quad k \leq n \\ 0, \text { for } k>n\end{array}\right.$

$\left(e_{n k}^{(r)}\right)$ is called the $(E, r)$ matrix.

Remark 1.1. Note that $r \neq 0$, since $|1-r|<1$.

The following results are needed in the sequel.

Theorem 1.2. ([9], Theorem 1.2) The (E,r) method is regular.

Theorem 1.3. ([9], Theorem 1.3) $\left(e_{n k}^{(r)}\right)\left(e_{n k}^{(s)}\right)$ is the $(E, r s)$ matrix.

Corollary 1.1. ([9], Corollary 1.4) The $(E, r)$ matrix is invertible and its inverse is the $\left(E, \frac{1}{r}\right)$ matrix.

\section{Main Results}

In this section, we prove some interesting properties of the Euler method. 
Theorem 2.1 (Limitation theorem). If $\sum_{k=0}^{\infty} x_{k}$ is $(E, r)$ summable, then $\left\{x_{k}\right\}$ is bounded.

Proof. Let $\left\{\sigma_{n}^{(r)}\right\}$ be the $(E, r)$ transform of $\left\{s_{n}\right\}$, where $s_{n}=\sum_{k=0}^{n} x_{k}$, $n=0,1,2, \ldots$, i.e.,

$$
\sigma_{n}^{(r)}=\sum_{k=0}^{n}{ }^{n} c_{k} r^{k}(1-r)^{n-k} s_{k}, \quad n=0,1,2, \ldots
$$

By hypothesis $\lim _{n \rightarrow \infty} \sigma_{n}=\sigma$ (say). So $\left\{\sigma_{n}\right\}$ is bounded, i.e., there exists $M>0$ such that $\left|\sigma_{n}\right| \leq M, n=0,1,2, \ldots$. Note that, in view of Corollary (1.1),

$$
\begin{gathered}
s_{n}=\sum_{k=0}^{n}{ }^{n} c_{k}\left(\frac{1}{r}\right)^{k}\left(1-\frac{1}{r}\right)^{n-k} \sigma_{k}, \quad n=0,1,2, \ldots \\
\text { Thus }\left|s_{n}\right| \leq \max _{0 \leq k \leq n}\left|{ }^{n} c_{k}\right| \frac{1}{|r|^{k}} \frac{|r-1|^{n-k}}{|r|^{n-k}}\left|\sigma_{k}\right| \\
\leq M,
\end{gathered}
$$

since $\left|{ }^{n} c_{k}\right| \leq 1,|r-1|<1,|r|=|(r-1)+1|=\max (|r-1|, 1)=1$. Consequently, $\left|x_{k}\right|=\left|s_{k}-s_{k-1}\right| \leq \max \left(\left|s_{k}\right|-\left|s_{k-1}\right|\right) \leq M, k=0,1,2, \ldots$ so that $\left\{x_{k}\right\}$ is bounded.

Remark 2.1. We recall that the classical Mazur-Orlicz theorem says that if a conservative matrix sums a bounded divergent sequence, then it sums an unbounded one. It was pointed out in [8] that the above theorem fails to hold in the ultrametric case, a counterexample being any regular $\left(N, p_{n}\right)$ method. Theorem (??) shows that any $(E, r)$ method is also a counter example to show that the Mazur-Orlicz theorem fails to hold in the ultrametric set up.

Definition 2.1. Given a sequence $\left\{x_{k}\right\}$, define the sequence $\left\{\bar{x}_{k}\right\}$ by

$$
\bar{x}_{0}=0, \quad \bar{x}_{k}=x_{k-1}, \quad k \geq 1 .
$$

$A=\left(a_{n k}\right)$ is said to be left translative if the $A$-summability of $\left\{x_{k}\right\}$ to $s$ implies the $A$-summability of $\left\{\bar{x}_{k}\right\}$ to $s . A$ is said to be right translative if the $A$ summability of $\left\{\bar{x}_{k}\right\}$ to $s$ implies the $A$-summability of $\left\{x_{k}\right\}$ to $s$. If $A$ is both left and right translative, $A$ is said to be translative.

Theorem 2.2. $(E, r)$ is translative. 
Proof. Let $\left\{\sigma_{n}(r)\right\}$ be the $(E, r)$ transform of $\left\{x_{k}\right\}$ and $\left\{\tau_{n}(r)\right\}$ be the $(E, r)$ transform of $\left\{\bar{x}_{k}\right\}$. We shall now prove that

$$
\begin{aligned}
\sigma_{n}(r) & =\left(1-\frac{1}{r}\right) \tau_{n}(r)+\frac{1}{r} \tau_{n+1}(r), \\
\text { i.e., } \sum_{k=0}^{n}{ }^{n} c_{k} r^{k}(1-r)^{n-k} x_{k} & =\left(1-\frac{1}{r}\right) \sum_{k=0}^{n}{ }^{n} c_{k} r^{k}(1-r)^{n-k} \bar{x}_{k} \\
& +\frac{1}{r} \sum_{k=0}^{n+1}(n+1) c_{k} r^{k}(1-r)^{n+1-k} \bar{x}_{k}, \\
i . e ., \sum_{k=0}^{n}{ }^{n} c_{k} r^{k}(1-r)^{n-k} x_{k} & =\left(1-\frac{1}{r}\right) \sum_{k=1}^{n}{ }^{n} c_{k} r^{k}(1-r)^{n-k} x_{k-1} \\
& +\frac{1}{r} \sum_{k=1}^{n}(n+1)
\end{aligned}
$$

For $0 \leq j \leq n$, coefficient of $x_{j}$ on the left side of $(2.1)={ }^{n} c_{j} r^{j}(1-r)^{n-j}$. Also coefficient of $x_{j}$ on the right side of (2.1)

$$
\begin{aligned}
& =\left(1-\frac{1}{r}\right){ }^{n} c_{(j+1)} r^{j+1}(1-r)^{n-j-1}+\frac{1}{r}{ }^{(n+1)} c_{(j+1)} r^{j+1}(1-r)^{n-j} \\
& =-\frac{1-r}{r}{ }^{n} c_{(j+1)} r^{j+1}(1-r)^{n-j-1}+\frac{1}{r}{ }^{(n+1)} c_{(j+1)} r^{j+1}(1-r)^{n-j} \\
& =-\frac{1}{r}{ }^{n} c_{(j+1)} r^{j+1}(1-r)^{n-j}+\frac{1}{r}{ }^{(n+1)} c_{(j+1)} r^{j+1}(1-r)^{n-j} \\
& =-{ }^{n} c_{(j+1)} r^{j}(1-r)^{n-j}+{ }^{(n+1)} c_{(j+1)} r^{j}(1-r)^{n-j} \\
& =\left\{{ }^{(n+1)} c_{(j+1)}-{ }^{n} c_{(j+1)}\right\} r^{j}(1-r)^{n-j} \\
& ={ }^{n} c_{j} r^{j}(1-r)^{n-j} .
\end{aligned}
$$

Thus (2.1) holds. Suppose $\lim _{n \rightarrow \infty} \tau_{n}(r)=s$. Taking limit as $n \rightarrow \infty$ in (2.1), we see that

$$
\lim _{n \rightarrow \infty} \sigma_{n}(r)=\left(1-\frac{1}{r}\right) s+\frac{1}{r} s=s,
$$

so that $(E, r)$ is right translative. Now,

$$
\tau_{n}(r)=\sum_{k=0}^{n}{ }^{n} c_{k} r^{k}(1-r)^{n-k} \bar{x}_{k}
$$




$$
\begin{aligned}
& =\sum_{k=1}^{n}{ }^{n} c_{k} r^{k}(1-r)^{n-k} x_{k-1}, \quad \text { since } \bar{x}_{0}=0 \\
& =\sum_{j=0}^{n-1}{ }^{n} c_{(j+1)} r^{j+1}(1-r)^{n-j-1} s_{j} \\
& =\sum_{j=0}^{n-1}{ }^{n} c_{(j+1)} r^{j+1}(1-r)^{n-j-1}\left(\sum_{k=0}^{j}{ }^{j} c_{k}\left(\frac{1}{r}\right)^{k}\left(1-\frac{1}{r}\right)^{j-k} \sigma_{k}(r)\right) \\
& =\sum_{k=0}^{n-1} r(1-r)^{n-k-1} \sigma_{k}(r)\left(\sum_{j=k}^{n-1}(-1)^{j-k}{ }^{n} c_{(j+1)}{ }^{j} c_{k}\right)
\end{aligned}
$$

That is $\tau_{n}(r)=\sum_{k=0}^{n-1} r(1-r)^{n-k-1} \sigma_{k}(r)\left(\sum_{j=k}^{n-1}(-1)^{j-k}{ }^{n} c_{(j+1)}{ }^{j} c_{k}\right)$.

Using the identity $\sum_{k=0}^{n-1}\left(\sum_{j=k}^{n-1}(-1)^{j-k}{ }^{n} c_{(j+1)}{ }^{j} c_{k}\right)^{z^{k}}=\sum_{k=0}^{n-1} z^{k}$, we see that

$$
\sum_{j=k}^{n-1}(-1)^{j-k}{ }^{n} c_{(j+1)}{ }^{j} c_{k}=1, \quad 0 \leq k \leq(n-1)
$$

In view of (2.2), we have, $\tau_{n}(r)=\sum_{k=0}^{n-1} r(1-r)^{n-k-1} \sigma_{k}(r)$.

Since $|1-r|<1$, all the conditions of theorem (1.1) are fulfilled and so $\lim _{k \rightarrow \infty} \sigma_{k}(r)=s$ implies that $\lim _{n \rightarrow \infty} \tau_{n}(r)=s$. Thus $(E, r)$ is left translative. This completes the proof of the theorem.

Definition 2.2. The infinite matrix methods $A=\left(a_{n k}\right), B=\left(b_{n k}\right)$ are said to be 'consistent' if no sequence is summable to different values by $A$ and $B$, i.e., if a sequence $\left\{x_{n}\right\}$ is $A$ summable to $\ell$ and $B$ summable to $m$, then $\ell=m$.

As in the case of regular $\left(N, p_{n}\right)$ methods (see [11], Theorem 4.1), we have the following result.

Theorem 2.3. Any two Euler methods are consistent. 
Proof. Consider the Euler methods $(E, r)$ and $(E, s)$. We then have $|1-r|$, $|1-s|<1$. Let $\left\{\sigma_{n}(r)\right\},\left\{\tau_{n}(s)\right\}$ be the $(E, r),(E, s)$ transforms of $\left\{x_{n}\right\}$ respectively. Let $\lim _{n \rightarrow \infty} \sigma_{n}(r)=\sigma$ and $\lim _{n \rightarrow \infty} \tau_{n}(s)=\tau$. We claim that $\sigma=\tau$.

Now, $\sigma_{n}(r)=(E, r)\left(\left\{x_{n}\right\}\right)$ and $\tau_{n}(s)=(E, s)\left(\left\{x_{n}\right\}\right)$.

$$
\text { So } \begin{aligned}
\sigma_{n}(r) & =(E, r)(E, s)^{-1}\left(\tau_{n}(s)\right) \\
& =\left((E, r)\left(E, \frac{1}{s}\right)\left(\tau_{n}(s)\right), \text { using Corollary }(1.1)\right. \\
& =\left(E, \frac{r}{s}\right)\left(\tau_{n}(s)\right), \text { using theorem }(1.3)
\end{aligned}
$$

Note that $\left|1-\frac{r}{s}\right|=\left|\frac{s-r}{s}\right|=|s-r|, \quad$ since $|s|=1$, using $|1-s|<1$

$$
\begin{aligned}
& =|(1-r)-(1-s)| \\
& \leq \max (|(1-r)|,|(1-s)|) \\
& <1,
\end{aligned}
$$

so that $\left(E, \frac{r}{s}\right)$ is regular, in view of definition (1.1) and theorem (1.2). Using (2.3), it follows that $\sigma=\tau$, completing the proof.

Remark 2.2. In view of Theorem 2.3, we are able to define a parameterless Euler method $E$ of summability as follows.

A sequence $\left\{x_{n}\right\}$ is summable $E$ to $\sigma$ if there exists $r \in K,|1-r|<1$ such that $\left\{x_{n}\right\}$ is $(E, r)$ summable to $\sigma$.

We shall now prove a few Tauberian theorems for the method $(E, r)$ modelled on those proved for $\left(N, p_{n}\right)$ methods by Natarajan [7].

Theorem 2.4. If $\sum_{k=0}^{\infty} a_{k}$ is $(E, r)$ summable to $\sigma$ and if $a_{n} \rightarrow \ell$, $n \rightarrow \infty$, then $\sum_{k=0}^{\infty} a_{k}$ converges to $\sigma$.

Proof. In view of theorem (1) of [7], it suffices to prove that the sequence $\{k\}$ of integers is not $(E, r)$ summable. Let $\left\{\sigma_{n}(r)\right\}$ be the $(E, r)$ transform of $\{k\}$, i.e., $\sigma_{n}(r)=\sum_{k=0}^{n}{ }^{n} c_{k} r^{k}(1-r)^{n-k} k, \quad n=0,1,2, \ldots$

$$
\text { Now, } \begin{aligned}
\sigma_{n+1}(r)-\sigma_{n}(r) & =\sum_{k=0}^{n+1}{ }^{(n+1)} c_{k} r^{k}(1-r)^{n+1-k} k-\sum_{k=0}^{n}{ }^{n} c_{k} r^{k}(1-r)^{n-k} k \\
& =\sum_{k=1}^{n+1}{ }^{(n+1)} c_{k} r^{k}(1-r)^{n+1-k} k-\sum_{k=1}^{n}{ }^{n} c_{k} r^{k}(1-r)^{n-k} k
\end{aligned}
$$




$$
\begin{aligned}
& =\sum_{k=1}^{n}{ }^{(n+1)} c_{k} r^{k}(1-r)^{n+1-k} k+r^{n+1}(n+1) \\
& -\sum_{k=1}^{n-1}{ }^{n} c_{k} r^{k}(1-r)^{n-k} k-r^{n} n
\end{aligned}
$$

Using $|1-r|<1,|r|=1,|k| \leq 1, k=0,1,2, \ldots$, we have,

$$
\begin{aligned}
\left|\sum_{k=1}^{n}{ }^{(n+1)} c_{k} r^{k}(1-r)^{n+1-k} k\right| & \leq \underset{1 \leq k \leq n}{\operatorname{Max}}\left|{ }^{(n+1)} c_{k}\right||r|^{k}|1-r|^{n+1-k}|k| \\
& <1
\end{aligned}
$$

Similarly, $\left|\sum_{k=1}^{n-1}{ }^{n} c_{k} r^{k}(1-r)^{n-k} k\right|<1$;

$$
\begin{aligned}
\left|r^{n+1}(n+1)-r^{n} n\right| & =\left|n r^{n}(r-1)+r^{n+1}\right| \\
& =\operatorname{Max}\left\{|n||r|^{n}|r-1|,|r|^{n+1}\right\} \\
& =1,
\end{aligned}
$$

so that $\left|\sigma_{n+1}(r)-\sigma_{n}(r)\right|=1, \quad n=0,1,2, \cdots$ Thus $\left\{\sigma_{n}(r)\right\}$ is not a Cauchy sequence and hence diverges, i.e., $\{k\}$ is not $(E, r)$ summable, completing the proof.

Using Theorem (3) of [7], we have,

Theorem 2.5. If $\sum_{k=0}^{\infty} a_{k}$ is $(E, r)$ summable to $\sigma$ and if $a_{n+1}-a_{n} \rightarrow \ell$, $n \rightarrow \infty$, then $\sum_{k=0}^{\infty} a_{k}$ converges to $\sigma$.

As in the case of regular $\left(N, p_{n}\right)$ methor ([7], Theorem 5), we have the following theorem too.

Theorem 2.6. If $\sum_{k=0}^{\infty} a_{k}$ is $(E, r)$ summable, then the following Tauberian conditions are equivalent:

(i) $a_{n} \rightarrow \ell, n \rightarrow \infty$;

(ii) $a_{n+1}-a_{n} \rightarrow \ell^{\prime}, n \rightarrow \infty$.

If, further, $a_{n} \neq 0, n=0,1,2, \ldots$, each of

(iii) $\frac{a_{n+1}}{a_{n}} \rightarrow 1, n \rightarrow \infty$; and

(iv) $\frac{a_{n+2}+a_{n}}{a_{n+1}} \rightarrow 2, n \rightarrow \infty$

is a weaker Tauberian condition for the $(E, r)$ summability of $\sum_{k=0}^{\infty} a_{k}$. 


\section{References}

[1] R.P. Agnew, Euler transformations, Amer. J. Math., 66 (1944), 313-338.

[2] G. Bachman, Introduction to p-adic numbers and valuation theory, Academic Press, 1964, zbl 0192.40103 MR 169847.

[3] G.H. Hardy, Divergent Series, Oxford University Press, 1949.

[4] A.F. Monna, Sur le théorème de Banach-Steinhaus, Indag. Math. , 25 (1963), 121-131, zbl 0121.32703 MR 151823.

[5] P.N. Natarajan, Criterion for regular matrices in non-archimedean fields, $J$. Ramanujan Math. Soc., 6 (1991), 185-195, zbl 0751.40003 MR 1132355

[6] P.N. Natarajan, On Nörlund method of summability in non-archimedean fields, J. Analysis , 2 (1994), 97-102,zbl 0807.40005 MR 1281500.

[7] P.N. Natarajan, Some Tauberian theorems in non-archimedean fields, $p$ adic functional analysis, Marcel Dekker, 192 (1997), 297-303.

[8] P.N. Natarajan, Failure of two classical summability theorems in nonarchimedean analysis, J. Analysis , 7 (1999), 1-5.

[9] P.N. Natarajan, Euler and Taylor methods of summability in complete ultrametric fields, J. Analysis , 11 (2003), 33-41.

[10] R.E. Powell and S.M. Shah, Summability theory and applications (Revised Edition), Prentice-Hall of India, 1988.

[11] V.K. Srinivasan, On certain summation processes in the $p$-adic field, Indag. Math., 27 (1965), 319-325, zbl 0128.28004 MR 196334. 